\title{
Il rococò a Valencia e la sua applicazione nell'adorno architettonico
}

\author{
Rococo in Valencia and his application in architectonic adorns
}

\author{
GaEtano GiannotTa \\ al377467@uji.es
}

Università degli Studi di Palermo

Universitat Jaume I

\begin{abstract}
Riassunto: Il Rococò si manifestò nella città di Valencia durante il trentennio centrale del Settecento e si espresse in tutte le forme dell'arte. Raggiunse l'apice della sua ricchezza nella decorazione degli interni, primi tra tutti quelli del palazzo de Dos Aguas e della vicina chiesa di San Andrés. Il suo successo cessò con l'avvento dell'Accademismo, che con l'istituzione della Reale Accademia di Belle Arti di San Carlos nel 1768, pretese il controllo delle arti sotto il segno del neoclassicismo. Si è sempre relegato il Rococò nelle ultime pagine dell'evoluzione del Barocco valenzano e manca uno studio indipendente del Rococò valenciano, delle sue fonti stilistiche, dei modelli che impiega, delle sue relazioni con gli stili precedenti, delle cause che determinano il suo trentennale successo. Questo articolo pretende iniziare a far luce su questi aspetti, confidando che approfondimenti futuri possano valorizzare le produzioni di uno dei periodi più ricchi della storia dell'arte valenciana.
\end{abstract}

Parole chiave: rococò, Valencia, XVIII secolo, accademismo.

\begin{abstract}
Rococo emerged in the city of Valencia in the central three decades of 18th century and it has been expressing itself in all forms of art. It reached the peak of its richness in interior decoration, first of all those of the palace de Dos Aguas and the nearby church of San Andrés. Its success ended because of arrival of Academism, with the establishment in 1768 of the Royal Academy of Fine Arts of San Carlos, which claims to control the arts under the sign of neoclassicism. The Rococo has been always relegated to the last pages of the evolution of the Valencian Baroque. Nowadays, an independent study of the Valencian Rococo and its stylistic sources, of the models it employs, of its relations with previous styles, of the reasons of its thirty-year success, is still missing. This article pretends to shed light on these aspects, trusting that further studies can enhance the products of one of the richest periods in the history of Valencian art.
\end{abstract}

Keywords: rococo, Valencia, XVIII century, academicism. 


\section{Introduzione}

Il Barocco a Valencia tradizionalmente si fa iniziare con la rinnovazione del presbiterio della Cattedrale di Valencia tra 1674 e 1682 e terminare con l'approvazione degli statuti che danno vita alla Real Academia de Bellas Artes de San Carlos il 12 febbraio del 1768 (Bérchez 1987: 203-216; Pingarrón 1998: 111-120). Stilisticamente questa fase lunga quasi un secolo fu ben lungi dall'essere unitaria. Si può suddividere in quattro momenti, fasi o stili, ma questa comoda divisione non è da considerarsi incontrovertibile né cronologicamente determinata. A regnare fu l'eterogeneità e spesso, mentre un determinato modello stilistico e formale era in auge, contemporaneamente ne nasceva e se ne affermava uno ben differente. Tuttavia. si può e si deve parlare ancora di un Barocco valenzano unitario, nella misura in cui si analizzano i basilari elementi comuni a tutte le citate fasi. Innanzitutto, il grande protagonismo del Barocco nella nuova definizione decorativa degli interni. Nel corso del Sei e Settecento, la maggior parte degli interni ecclesiastici e privati della città furono letteralmente rivestiti da una nuova ed esuperante pelle barocca che in molti casi è quella con la quale si presentano oggi agli occhi dello spettatore (González Tornel 2008: 114117; Pingarrón 1997: 327). In secondo luogo, non cessa la dipendenza dai temi controriformisti, dall'esaltazione dell'Eucarestia all'importanza della Catechesi, dall'apologia al culto mariano al sostegno nei confronti del Papato.

Ma soprattutto non deve dimenticarsi la strenua difesa del culto ai santi, titolari di templi che si andarono rinnovando per mezzo di programmi iconografici complessi basati sull'esaltazione della loro vita e delle virtù da loro perseguite (Pingarrón 1998: 67-73). Infine, con certo interesse deve segnalarsi il sorgere di un nuovo mecenatismo di tipo aristocratico e profano, che non sostituisce, ma si affianca a quello ecclesiastico. Molti furono gli aristocratici che rinnovarono i loro palazzi secondo il nuovo gusto barocco e che non raramente incisero nelle numerose restaurazioni ecclesiastiche realizzate durante il secolo in esame, in forza delle loro relazioni con centri culturali stranieri, soprattutto italiani (Bérchez 1993: 130-132). Conseguenza palese di quanto si è detto fu che il volto della città di Valencia cambiò radicalmente, come si può apprezzare anche solo paragonando le piante della città realizzate negli anni a cavallo tra i due secoli: il piano di Mançeli del 1695, il più antico della città mai realizzato, e il più famoso piano del padre oratoriano Tomás Tosca del 1702 (Pingarrón 1998: 20-22).

\section{L'evoluzione del Barocco valenzano verso il Rococò}

La prima fase stilistica del Barocco valenzano è quella che si aprì con il già citato rinnovamento del presbiterio della Cattedrale a partire dal 1674. È quel momento che Elías Tormo definì barroco castellano e che secondo lo studioso valenzano derivava dal churriguerismo (Tormo y Monzó 1920a: 123; 1921: 208-210). Stilisticamente si caratterizzò per una profusione decorativa senza precedenti nell'ambiente artistico valenzano. Elementi decorativi di carattere soprattutto vegetale riempirono 
gli antichi spazi architettonici riformati in questa fase in risposta al diktat estetico del barocco horror vacui. Si rivestirono principalmente i retabli, le cornici di finestre e le superfici di pilastri, colonne e archi delle volte. Non mancarono anche figure a tutto tondo, soprattutto i carnosi putti e angeloni con funzione di telamoni sotto le cornici delle volte. È manifesta però una certa ripetitività, quasi una realizzazione in serie di questi elementi (González Tornel 2008: 117-121). Iconograficamente poi, non aggiungevano nulla o aggiungevano veramente poco al messaggio d'insieme dei templi rinnovati, essendo la funzione iconologica riservata ancora e tradizionalmente a pitture e gruppi scultorei (Vilaplana Zurita 1997: 95-123). Tradizionali continuavano ad essere anche i processi d'esecuzione, i materiali impiegati e le modalità di assegnazione delle commesse. Si impiegavano soprattutto il marmo e il gesso per gli elementi in rilievo e a tutto tondo, mentre per la decorazione piana si proseguiva nell'impiegare la tecnica secentesca dell'esgrafiado (Ferrer Orts 2000: 105-109).

Le norme corporative controllavano l'assegnazione ed organizzazione dei lavori: l'obrer de vila, vero e proprio architetto in senso moderno, ideava il progetto, si aggiudicava le commesse e ne subappaltava l'esecuzione; i pedrapiquers e gli albañiles, che si possono equiparare ai moderni tagliapietre e carpentieri, realizzavano le opere architettoniche in pietra, i carpinteros quelle in legno; un braccio di questi ultimi era costituito da retablistas, pittori e scultori che modellavano le decorazioni e le opere mobili dei congiunti architettonici. Però tutti dovevano in qualunque caso sottomettersi al progetto del obrer de vila o maestro d'opera, che era l'unico a possedere le conoscenze e capacità tecniche per adempiere l'opera (González Tornel 2005a: 14-15, 304). Figura chiave del Barroco castellano fu l'architetto Juan Bautista Pèrez Castiel (†1734) il cui progetto di rinnovamento del presbiterio della Cattedrale di Valencia introdusse per la prima volta nella città il Barocco, secondo l'analisi realizzata da Fernando Pingarrón (1998: 109-120; De Orellana 1967: 523-524). Realizzò quest'opera tra il 1674 e il 1682 impiegando tutti gli elementi stilistici e tecnici che si sono elencati qualche riga fa.

Forse per questa ragione e per il successo che le sue opere ottennero, la prima fase del Barocco valenzano è nota nei manuali anche come estilo perezcasteliano. Per citare solo alcuni principali esempi, sono attribuibili a questa fase castellana o perezcasteliana del proto-barocco valenzano, oltre il più volte citato presbiterio cattedralizio, il presbiterio dell'Arcipretale di Morella, l'Arcipretale di Chelva, la Chiesa parrocchiale di Tuejár, la Chiesa del Salvatore di Requena, la Cappella della Comunione della Chiesa dell'Assunzione di Biar, la Cappella della Comunione della Chiesa parrocchiale di Onil, la Chiesa parrocchiale di Meliana, la Chiesa di San Pietro di Albalat de la Ribera, la chiesa del Monastero di Simat de la Valldigna, la chiesa del Convento di San Domenico di Orihuela, il Municipio di Castellón de la Plana, la Chiesa di Sant'Anna di Segorbe, la Galleria dorata del Palazzo ducale di Gandía, la Cappella di Santa Barbara della Chiesa di San Juan del Hospital di Valencia, e ancora nella capitale, il Collegio di San Pio V, la Chiesa di Santo Stefano, la chiesa del Monastero della Trinità, la Chiesa di Sant'Antonio, la torre campanaria di Santa Caterina, ecc. (Bérchez 1993: 26-68; Tormo y Monzó 1920a: 124-129). 
La seconda fase del Barocco valenzano fu quella italianizzante, che ebbe inizio con il rinnovamento dell'antico tempio gotico de los Santos Juanes o San Giovanni del Mercato tra 1693 e 1702 (González Tornel 2008: 114-117). È l'opera che più rappresentò, in pieno cambio di secolo, il passaggio verso concezioni, tecniche e stili più vicini alla situazione artistica italiana. Questo venne causato dalla contemporanea presenza nella città di artisti italiani, in particolare lo scultore cremonese Giacomo Bertesi (1643-1710) e lo stuccatore comasco Antonio Aliprandi (1654-1728), o formatisi nel medio artistico italiano, come l'architetto tedesco Conrad Rudolph (†1732).

I primi due artisti citati parteciparono attivamente nella riferita decorazione dell'interiore de los Santos Juanes sulla base di un programma iconografico ideato dal canonico Vicente Vitoria (1650-1709), pittore e intellettuale strettamente connesso all'ambiente culturale di Firenze e Roma, dove aveva trascorso buona parte della sua vita e dove si era formato artisticamente. Vitoria progettò per los Santos Juanes un complesso programma finalizzato all'esaltazione delle virtù dei santi titolari della chiesa tramite la relazione tra statue allegoriche, ovali pittorici con scene della loro vita e un immenso e grandioso affresco realizzato da Antonio Palomino (16551726) con la rappresentazione dell'Adorazione dell'Agnello mistico nella volta del presbiterio e il Trionfo della Chiesa in quella della navata (González Tornel 2005a: 145-148; Vilaplana Zurita 1997: 294-295).

Los Santos Juanes fu il trionfo del dogma estetico del Barocco che aspirava alla commistione e alla intima compenetrazione di tutte le arti (Bérchez 1993: 132; Tormo y Monzó 1920a: 120). Stilisticamente si assistette all'introduzione di elementi assolutamente innovativi, come le statue distese sugli archi di accesso alle cappelle laterali, quelle esenti su mensole nei contrafforti, gli ovali pittorici, che ricordano precedenti esperienze romane o fiorentine (Pingarrón 1998: 221-226; Vilaplana Zurita 1997: 277-278). Non si rinunciò alla profusione di più classici elementi decorativi di tipo vegetale, nella realizzazione dei quali un ruolo di primaria importanza assunse lo stuccatore Aliprandi. Tuttavia, tali elementi assunsero per la prima volta un taglio ben più naturalistico e realistico rispetto a quelli perezcasteliani, manifestando da parte del loro autore un attento studio del naturale mediante il disegno. Lo studio del naturale e l'uso del disegno e di modelli in terracotta vennero per la prima volta introdotti a Valencia proprio da questi artisti, dando il via ad innovazioni tecniche, certamente non prive di ragioni estetiche, che ebbero ripercussioni nelle fasi successive, soprattutto negli anni di formazione dell'Accademia di Santa Barbara, di cui si parlerà (González Tornel 2005: 308-310).

Mentre la decorazione diventava protagonista, l'architettura iniziò ad essere concepita e rinnovata in funzione di essa o non più al contrario; per la prima volta l'architettura risultò in certa misura addirittura sottomessa all'adorno. Cambiò poi il modo stesso di gestire le commissioni artistiche: a partire dagli anni di passaggio tra $\mathrm{i}$ due secoli, furono direttamente gli scultori e adornatori a progettare le proprie opere, interferendo di volta in volta sempre di più nell'ambito strettamente architettonico. Ancora una volta il comasco Aliprandi esemplificò perfettamente questo 
cambiamento: in uno dei suoi capolavori valenzani, il rivestimento in stucco della Cappella di San Pietro nella Cattedrale, egli stesso fornì i disegni per le sue spettacolari decorazioni e definì sé stesso adornista e estucador, contravvenendo a qualunque norma corporativa vigente (González Tornel 2002: 138-139; Pingarrón 1998: 123-128). Infine, ben differenti rispetto al passato furono le finalità iconologiche di questi complessi decorativi. Infatti, fu quello il periodo in cui si produsse a Valencia il maggior numero di programmi e cicli iconografici dall'elevatissimo valore semantico, oltre che strettamente formale, come analizzato da David Vilaplana Zurita nella sua tesi dottorale (Vilaplana Zurita 1997). Si pensi, a parte che alle già citate rinnovazioni della Chiesa de los Santos Juanes e della Cappella cattedralizia di San Pietro, alla facciata principale della stessa Cattedrale, al maestoso rivestimento della chiesa di San Nicola di Bari e San Pietro Martire, al presbiterio della chiesa del Monastero della Vergine del Miracolo di Cocentaina (Bérchez 1993: 69-87).

L'assimilazione delle innovazioni introdotte dagli artisti stranieri che soggiornarono a Valencia negli anni di transizione tra i due secoli da parte degli scultori ed architetti autoctoni, diede vita ad una nuova vertente dell'evoluzione dell'arte barocca valenziana, che si può definire «classica». Cronologicamente ci si trovava in un periodo di vero e proprio fermento culturale, storicamente e culturalmente coincidente con il cambio dinastico che seguì alla Guerra di Successione, con l'ascesa dei Borboni diede vita a una Monarchia di taglio illuminista. Da un lato, si assistette al sorgere di un circolo di intellettuali vincolati al mondo delle Matematiche e della Geometria.

I membri del cosiddetto gruppo de los novatores non di rado si intromisero nel fare artistico della città di Valencia, conducendolo lontano dagli eccessi del Barocco puramente ornamentale verso idee che si possono definire proto-neoclassiche (Bérchez 1993: 92-94; González Tornel 2005a: 67-88). Dall'altro, si affermarono quelle accademie e scuole private d'arte che si erano formate nel corso del secolo precedente e nel seno delle quali si formarono tutti i principali protagonisti artistici del periodo. Concettualmente queste scuole erano un'eredità delle innovazioni della fase precedente, posto che in esse l'insegnamento si basava sulla copia dal naturale e sull'impiego del disegno (Bérchez 1987: 61-63; De Orellana 1985-1987: 12-13). In uno di questi studi privati, quello della famiglia di origine genovese dei Capuz, si formò Francisco Vergara «il maggiore» (1681-1753) che può considerarsi come il maggiore rappresentante del Barocco classico.

De Orellana scrisse di lui che lavorava con uguale successo materiali molto diversi, secondo quanto aveva appreso lavorando con gli artisti stranieri come Rudolph, Aliprandi, Bertesi e Bussy (1967: 595-598). Si aggiunga che praticava in egual misura sia l'architettura, che la scultura, la pittura e la decorazione (González 2005b: 41-51; Pingarrón 1997: 331-332). Il suo intervento nel rivestimento della Chiesa dei Santi Martino Vescovo e Antonio Abate si può considerare esemplificativo di quello che si è definito barroco clásico valenciano. Tra il 1735 e il 1753 la struttura gotica dell'antico tempio fu letteralmente rivestita per mezzo di una nuova pelle di stucco bianco e dorato; le colonne addossate ai contrafforti furono decorate con rocaille nell'imoscapo, ricordando quelle della facciata della vicina Cattedrale; sopra gli archi di ingresso delle cappelle ovali pittorici raccontano scene 
agiografiche dei santi titolari della chiesa, come a inizio secolo era stato fatto ne los Santos Juanes; nel fregio, all'interno di classiche metope alternate a triglifi, si inserirono rilievi con gli Attributi della Chiesa, direttamente dipendenti da quelli della Cattedrale, dove adempivano la medesima funzione doppiamente architettonica e iconografica (Pingarrón 1998: 245-263). Dunque, dal punto di vista stilistico si assistette alla diretta applicazione delle novità introdotte dagli artisti stranieri che avevano lavorato a Valencia nei primi anni del secolo, però mediante una voluta semplificazione dell'ornamento, che non occultava più completamente le strutture architettoniche, ma le marcava elegantemente. Si era dunque difronte ad una sorta di «ritorno all'ordine», che anche i trattatisti dell'epoca, padre Tosca e Antonio Ponz in testa, non mancarono di incentivare (Bérchez 1993: 8892). Sul fronte iconografico, continuò la relazione tra pitture e rivestimento architettonico, secondo l'estetica barocca del continum delle arti.

Oltre alla citata Parrocchia di San Martino, possono considerarsi parte di questo momento artistico la rinnovazione della Chiesa di Santa Caterina, l'antica chiesa del Convento dell'Oratorio di San Filippo Neri, la Chiesa di San Sebastiano, la chiesa del Collegio di San Pio V, la Cappella dei Pescatori della Chiesa di San Andrés, la Cappella dell'Università, e, fuori dalla Capitale, la Chiesa parrocchiale di Alcalá de Chivert, la Cappella della Comunione della Basilica di Santa Maria di Elche, la Chiesa di Sant'Andrea de L'Alcúdia, la Chiesa parrocchiale di Albalat dels Sorrells, ecc. (Bérchez 1993: 88-125).

\section{Le basi estetiche e stilistiche del Rococò a Valencia}

Giusto nell'anno in cui a Valencia si concludeva la riforma della Chiesa di San Martino, nel 1753, avvenne un fatto di primaria importanza per l'evoluzione del Barocco valenzano verso concezioni stilistiche rococò: il sorgere dell'Accademia di Santa Barbara. La Academia de Pintura, Escultura y Arquitectura de Santa Bárbara vide la luce il 7 gennaio di quel fortunato anno. L'iniziativa di dotare la città di una accademia di gestione pubblica, come era stato fatto l'anno precedente nella Corte con l'istituzione della Academia de San Fernando, nacque dal desiderio di artisti che si erano formati culturalmente e artisticamente nella fase precedentemente descritta: i fratelli Ignacio e José Vergara, figli del citato Francisco «il maggiore», Luis Domingo e Cristobal Valero richiesero alle Loro Maestà Fernando VI e Maria Barbara di Braganza l'appoggio economico per favorire le Arti a Valencia. Gli stessi artisti sostennero economicamente la scuola per i suoi primi anni di vita e ne diventarono direttori nelle rispettive classi artistiche, insieme a Pasqual Miguél e Jaime Molins per l'Architettura (Bérchez 1987: 19-41). L'istituzione però ebbe vita davvero breve, posto che già nel 1761 si dovette chiudere per mancanza di fondi. Tuttavia, le ragioni per le quali si tentò la fondazione di una accademia delle arti e le modalità dell'insegnamento che si impartivano in quella di Santa Barbara, informazioni che conosciamo grazie alla Breve Noticia... pubblicata del 1757, costituiscono una fonte essenziale per comprendere la particolarità dell'ultimo momento del Barocco valenzano, appunto il Rococò. 
Di fatto, ci si ritrovava davanti a un modello di accademia che non era altro che una eredità delle accademie barocche del XVII secolo. Queste, perlopiù private, si basavano sullo studio del naturale per mezzo del disegno, concepito come centro di tutte le arti e del carattere liberale delle stesse. L'intima conoscenza del disegno e delle sue applicazioni consentiva agli artisti di questo primo accademismo valenzano di mantenere quella multidisciplinarità che è propria del Barocco e ancora di più del Rococò a Valencia. Così, per citare un esempio soltanto, Pasqual Miguél e Jaime Molins, un pittore prospettico e uno scultore di retabli rispettivamente, poterono insegnare ai giovani discepoli dell'Accademia di Santa Barbara l'Architettura. Si comprendono in questo modo le parole di Bérchez, quando scrisse: «Una cosa sería el empeño académico sin más, y otra muy distinta el academicismo ilustrado con base estética en el clasicismo como alternativa al barroco» (1987: 20).

Nel caso dell'Accademia di Santa Barbara l'impegno accademico «sin más» consisteva nella rigida schematizzazione degli insegnamenti e delle nozioni da impartire, ma si escluse qualunque rinnovamento in senso estetico o qualsivoglia apologia sulla superiorità di un'arte sull'altra, aspetti che invece saranno fondanti dell'Accademia intesa in senso illuministico. Lo chiariva bene la citata Breve Noticia... (1757: 27):

Porque assí como en las Universidades Literarias, que tienen este nombre por tener muchos Maestros sabios, y muchos Discípulos aplicados, es donde mejor se enseñas las Ciencias, assí también en las Academias es donde mejor se enseñan las Bellas Artes. Fuera de ellas es arbitraria, e irregular la enseñanza. Por lo común se meten á Maestros los que menos saben, y quizás estos son los que tienen más Discípulos, porque enseñan más a prisa que los otros: con que en lugar de mejorarle, se deterioran las artes. En las Academias se enseñan por principios, de espacio, con regularidad y método.

Nell'istituzione dell'Accademia di Santa Barbara c'era dunque una intenzione di organizzare e regolarizzare gli insegnamenti artistici, superando la rigidezza dell'apprendistato presso il maestro secondo le ormai stanche norme corporativistiche. Tutto questo non si tradusse in una rinuncia di quelle che erano state le concezioni stilistiche e formali in cui i Vergara o Domingo si erano formati. Per questa ragione, sebbene si poté parlare di proto-accademismo e di organizzazione accademica dell'insegnamento dell'Arte, non si poté però parlare di superamento del Barocco. Anzi, al contrario, giusto a partire dagli anni centrali del secolo la ricchezza degli elementi decorativi, la ricerca di una commistione tra le diverse arti, la volontà di veicolare un complesso messaggio iconologico, aspetti propri di tutte le fasi fin qui analizzate, si esasperarono, direi addirittura si complicarono, fino a un apice che mai era stato raggiunto e mai sarebbe stato superato.

Tale esasperazione ha inizio negli anni Quaranta del secolo con la riforma del Palazzo del Marchesato de Dos Aguas per iniziativa del terzo marchese, Giner Rabassa de Perellós. Della prima vera grande rinnovazione rococò della città di Valencia si può dire davvero poco, in quanto tra 
1854 e 1867 venne cancellata da una ulteriore riforma che, nel pieno eclettismo architettonico tipico dell'epoca, unì elementi neobarocchi, neorococò, neoimpero e orientali (Coll Conesa 2005: 81-89). Secondo De Orellana, il progetto della riforma settecentesca fu affidato a Hipólito Rovira (1695-1765) (1967: 335-339). Confermò questa attribuzione Elías Tormo, il quale ebbe a scrivere: «El genial pintor Hipólito Rovira, protegido de la casa, y que acabó en loco, dio la pauta general de la especialísima decoración rococó y las de la gran puerta y de las ventanas, reservándose la pintura total de las fachadas» (1923: 106). Definito «el romántico de nuestro rococó», Rovira fu soprattutto incisore e pittore, protetto del Marchese che lo ospitò nel suo palazzo dopo il suo ritorno da un decennale viaggio di formazione artistica a Roma, sul quale sfortunatamente poco si sa, a parte che aveva lavorato nei circoli di Conca e Giaquinto (Tormo y Monzó 1920b: 174-188; 1921: 201-204). Per realizzare la riforma del Palazzo de Dos Aguas Rovira dispose di due degli artisti più distaccati del momento, Ignacio Vergara e Luis Domingo.

Si trattava esattamente degli stessi artisti che una decina di anni dopo la riforma del palazzo marchesale, insieme al pittore José Vergara, istituirono l'Accademia di Santa Barbara, della quale furono professori di Scultura. Di conseguenza, non si può negare il ruolo che tale riforma palatina ebbe nell'affermarsi di quelle concezioni estetiche che sarebbero soggiaciute alla nascente Accademia di Santa Barbara. Come si accennava, poco rimane di tale riforma, ma quello che sopravvive ci mostra il sorgere e l'affermarsi di certo sperimentalismo e della stravaganza rococò durante quello che voleva essere, in senso completamente contrario, un periodo di ritorno all'ordine dopo gli eccessi del Barocco ornamentale. Sono due gli spazi dell'attuale Museo Nazionale della Ceramica a cui ci si riferisce: il maestoso portale d'ingresso al palazzo dall'attuale Calle de la Cultura e la volta di quello che era lo scalone d'onore.

In entrambi i casi si è davanti ad un'opera che vede la collaborazione di Rovira e di Ignacio Vergara e l'uso da parte dei due artisti di materiali e tecniche diverse, segno di quella multidisciplinarietà che costituisce il carattere più innovativo del Rococò valenzano. Inoltre, si assiste in entrambe le opere a una profusione senza precedenti di elementi allegorici e metaforici, il cui significato in alcuni casi è ancora lontano dall'essere interpretato. Il portale, definito da Tormo «cosa única en su género en el mundo», venne progettato da Rovira (in verità si conosce anche un disegno di Luis Domingo) e scolpito da Vergara in pietra alabastrina proveniente dalle cave di Niñerola di proprietà del Marchese di Dos Aguas (Aldana Fernández 1976: 89-97; Coll Conesa 2005:17-20; Tormo y Monzó 1920a: 119; Figura 1). 
Gaetano Giannotta. Il rococò a Valencia e la sua applicazione nell'adorno architettonico

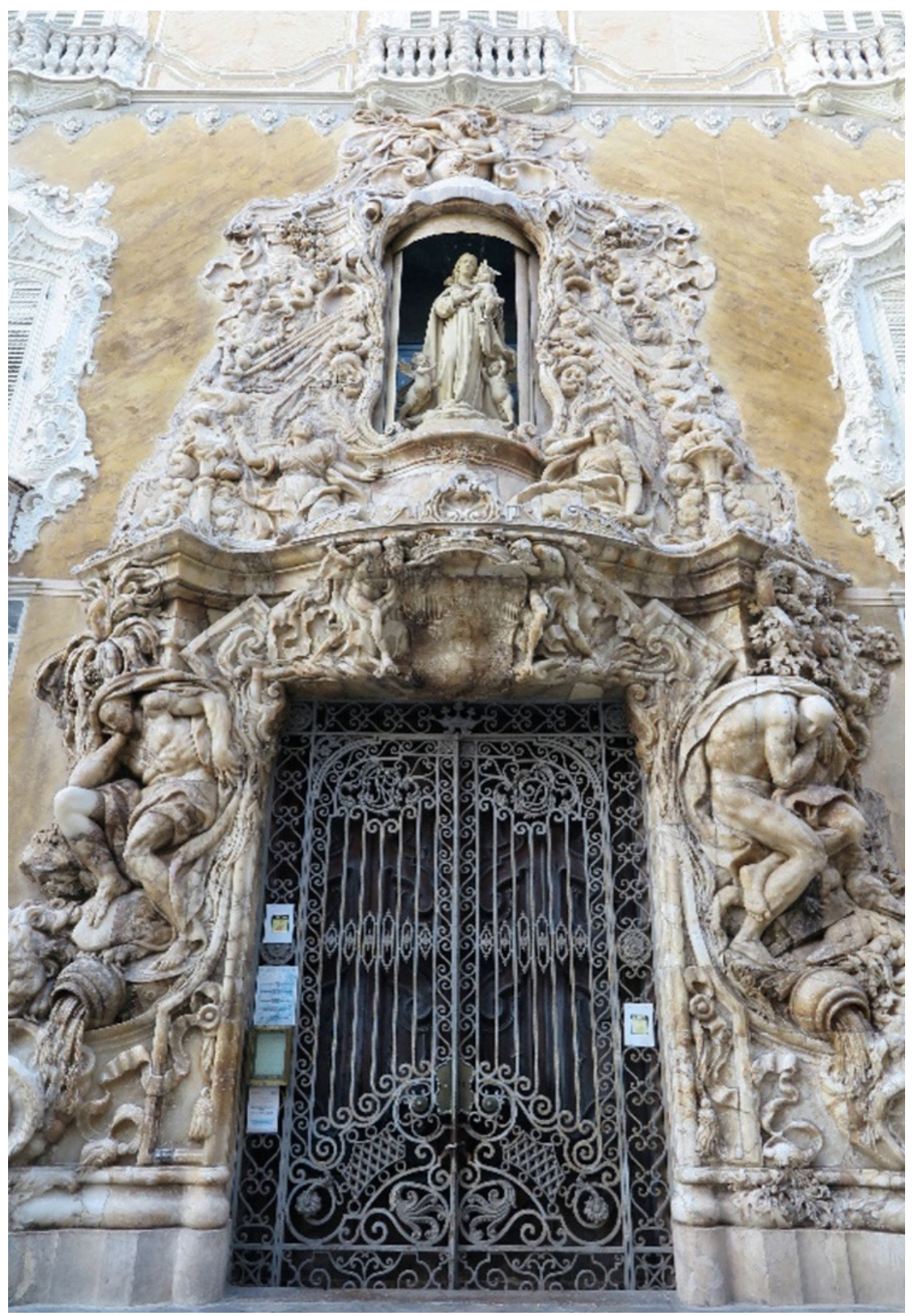

Figura 1 Ignacio Vergara scultore, Hipólito Rovira progettista, Portale principale, Museo Nazionale di Ceramica «González Martí», già Palazzo del Marchese de Dos Aguas, Valencia, ca.1740. Fotografia dell’autore.

SCRIPTA, Revista internacional de literatura i cultura medieval i moderna, núm. 14 / desembre 2019 / pp. 108-130 ISSN: 2340-4841 $\cdot$ doi:10.7203/SCRIPTA.14.16360 
Era la prima volta che lo scultore impiegava questo materiale, ma lo fece con un esito a dir poco straordinario. Stilisticamente si svincola totalmente da qualunque cosa fatta prima d'allora a Valencia, dove nel corso del Sei e Settecento si erano diffuse ed affermate tipologie di facciate che riproponevano le strutture dei monumentali retabli che si trovavano all'interno delle chiese. Dei modelli precedenti il portale del Palazzo marchesale di Dos Aguas mantiene solamente il carattere di struttura indipendente che pare aggiunta alla facciata. Tuttavia, qualunque elemento architettonico è scomparso, ad eccezione di una debole cornice che è comunque quasi completamente sopraffatta dalla decorazione. Questa, poi, è imprescindibile, costituisce il vero senso dell'opera e non, come in passato, un mero strumento riempitivo. Sopra una base di volute e rocaille due grandi sculture, una per ogni lato, rappresentano i due fiumi da cui il marchesato di Dos Aguas prende il proprio nome, il Jucar e il Magro. Sopra la cornice della porta, tra due raffigurazioni di Eracle con la sua mazza e la sua pelle di leone, si impianta lo stemma del marchesato. È la volta della cornice, sopra la quale si trova la nicchia con la Vergine del Rosario tra volute, conchiglie rugose, cornucopie, personaggi allegorici, ecc. Conclude l'insieme la figura della Fama alata in volo.

La decorazione è delirante e ancora maggiore doveva essere l'effetto d'insieme con le pitture della facciata realizzate dal poliedrico Rovira. Si percepisce l'intenzione di Vergara di tradurre tridimensionalmente in scultura una composizione pittorica o, ancor meglio, una incisione, arte nella quale Rovira, progettista come si è detto dell'opera, era versato. Stilisticamente è stato osservato come alcuni elementi siano derivati quasi direttamente dal Bernini, le cui opere Rovira poté ammirare durante il suo soggiorno romano, portando con sé al suo rientro in terra ispanica disegni e stampe. Ad esempio, i due grandi telamoni ai fianchi della porta deriverebbero dalle statue del Nilo e del Rio de la Plata della Fontana dei Quattro fiumi del Bernini a Roma, con le quali condividono anche gli attributi rispettivamente del leone, simbolo del continente africano, e del caimano, simbolo dell'America (Pérez Guillén 2008: 65-80).

Altre figure allegoriche, alcune di difficile interpretazione, decorano il portale. Oltre alla citata Fama alata che domina dall'alto il congiunto, ai lati della nicchia della Vergine si trovano le nereidi, ai suoi piedi sono le allegorie, derivate da Casare Ripa, del Buon Governo e dell'Agricoltura (1603: 9-10, 194), il tutto disposto simmetricamente tra una profusione senza eguali di rocaille, cornucopie ricche di frutti, nastri, altari infiammati e fumanti di incenso, palme, raggi di luce, nappe e volute. Al di là del significato iconografico del congiunto, più volte investigato (Pérez Guillén 2008; Sebastián López 1989; Vilaplana Zurita 1997), si vuole sottolineare come il portale del Palazzo de Dos Aguas costituì, grazie all'inusuale e delirante profusione di elementi decorativi, un vero e proprio vocabolario del Rococò valenzano che, come vedremo, fu parecchio sfruttato negli anni successivi. Il secondo elemento sopravvissuto alla riforma eclettica del Palazzo di Dos Aguas è la volta circolare di quello che era lo scalone d'onore (Figura 2). 


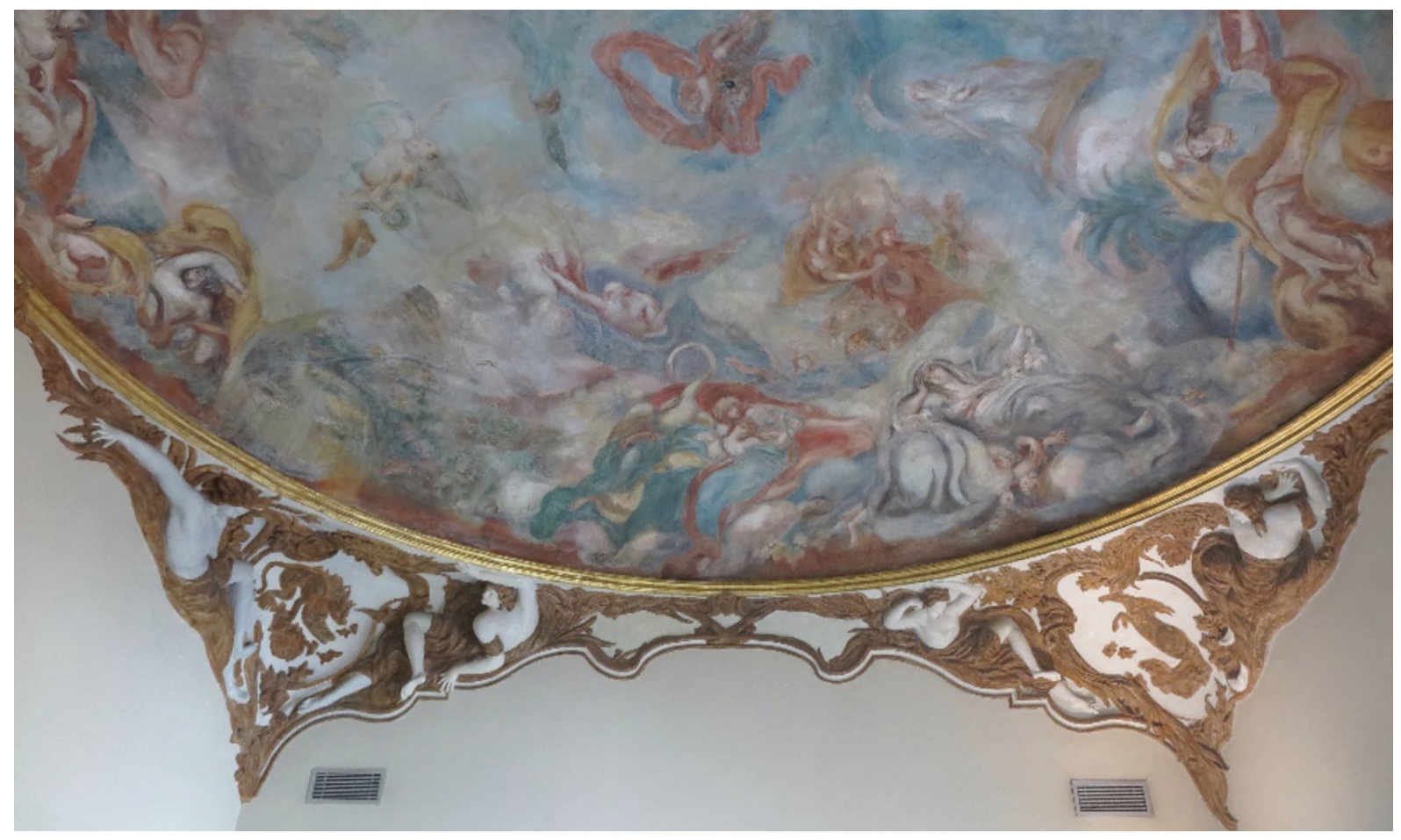

Figura 2 Ignacio Vergara scultore, Hipólito Rovira pittore e progettista, Cupola dello scalone d'onore,

Museo Nazionale di Ceramica «González Martí», già Palazzo del Marchese de Dos Aguas, Valencia, ca. 1740. Fotografia dell'autore.

La pittura centrale, realizzata da Rovira, rappresenta un insieme di figure allegoriche e mitologiche lungi dall'essere mai state interpretate, anche a causa del cattivissimo stato di conservazione. Nei pennacchi della volta Ignacio Vergara modellò in stucco gli animali simboleggianti i quattro continenti, recuperando il tema iconografico che in parte era stato introdotto nel portale con la raffigurazione dell'Africa e dell'America. Un elefante, un caimano, un cavallo e un leone entro sinuosissime cornici simboleggiano rispettivamente Asia, America, Europa e Africa, affiancati da coppie di eleganti telamoni che in pose differenti sorreggono l'insieme. La riforma del Palazzo de Dos Aguas che si è descritta mediante i resti che ne sopravvivono fu una riforma totalizzante, che letteralmente investì con il suo delirio decorativo ogni ambiente, compreso il suo contenuto. Questo, si è detto più volte, fu conseguenza del senso estetico stesso del Rococò valenzano, che tese a disintegrare qualunque residua barriera di distinzione tra le arti, mescolando architettura, scultura, pittura, incisione, tecniche e maestranze in un unico ed esuberante insieme iconologico. Si spiega allora come sia stato possibile che gli stessi artisti impegnati nella riforma architettonica del 
palazzo si siano occupati anche di realizzare qualcosa che prescindesse dall'architettura così come dai materiali che impiegavano normalmente: la Carrozza delle Ninfe (Figura 3).

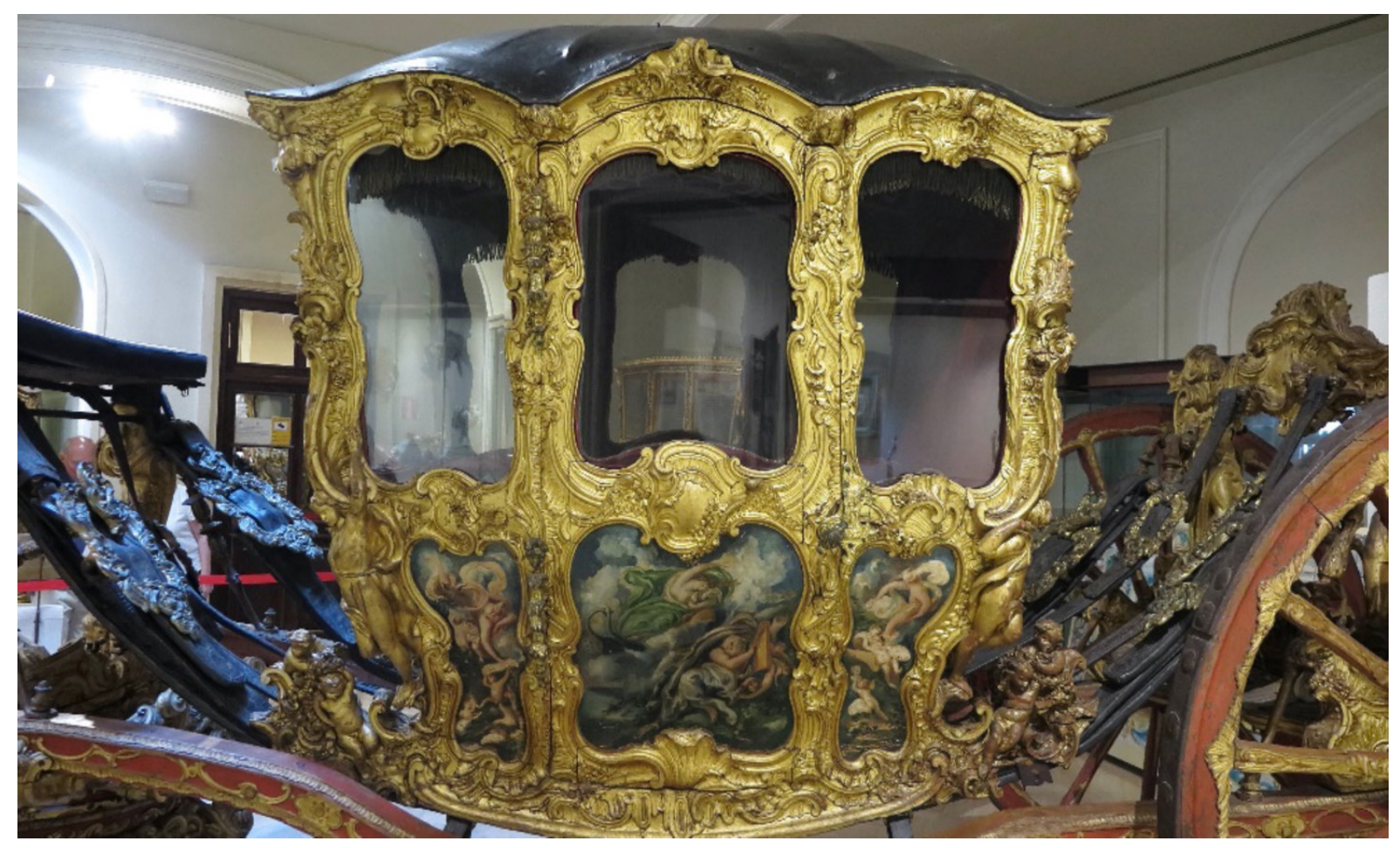

Figura 3 Ignacio Vergara intagliatore, Hipólito Rovira pittore, Carrozza delle Ninfe, Museo Nazionale di Ceramica «González Martí», già Palazzo del Marchese de Dos Aguas, Valencia, 1753. Fotografia dell'autore.

Forse la più rococò tra le carrozze che la Storia dell'Arte ricorda, la Carrozza delle Ninfe fu progettata da Ignacio Vergara che, per intagliarla, fu coadiuvato da Luis Domingo e altri scultori, mentre le eleganti pitture della cassa furono realizzate da Hipólito Rovira (Coll Conesa 2005: 4345). Tanto stilisticamente quanto iconograficamente la carrozza sembra quasi un prolungamento del palazzo stesso, con la sua abbondanza di nereidi, telamoni, rocaille, ghirlande floreali, teste leonine, elementi insomma che si sono rintracciati già nel portale di accesso al palazzo e nella volta del suo antico scalone d'onore e che ritroveremo, diversamente declinati e con altri materiali, nelle opere che si analizzeranno a seguire. Si è detto «iconograficamente» e lo si è detto con coscienza, perché nel Rococò valenzano il compito di trasmettere un messaggio iconologico non si riservava più solo ai ricchi retabli o congiunti scultorei delle chiese, né solamente ai programmi pittorici delle 
gallerie palatine. Ogni cosa, dal ricchissimo portale d'alabastro alla non meno preziosa carrozza di rappresentanza, doveva comunicare e doveva farlo stupendo e sopraffacendo con la sua esuberanza e abbondanza (Milam Dawn 2011: 23).

\section{La riforma della Chiesa di San Andrés: il più ricco esempio di Rococò valenzano}

In mancanza della documentazione che attesta la sua contrattazione, si può soltanto congetturare sulle fasi della barocchizzazione della chiesa di San Andrés, che più di ogni altra riforma tra quelle attuate fino ad allora a Valencia rappresentò il vero paradigma di come il Rococò non sia stata soltanto una corrente di passaggio e di come abbia lasciato nella città ben più che pochi sporadici e casuali esempi. L'antico tempio parrocchiale, dedicato da Giacomo I al santo patronimico del suocero Andrea II d'Ungheria che tanto lo aveva sostenuto economicamente nella Riconquista di Valencia, sede della Confraternita dei Pescatori dei quali Sant'Andrea era il protettore, era stato riedificato nel XVII secolo in base a stanche concezioni tardo-classiciste (Bérchez 1983: 514-522; Gómez-Ferrer Lozano 1995: 235-258). Così alla metà del Settecento si decise di dotare la chiesa di un nuovo rivestimento che più e meglio si confacesse al nuovo gusto stilistico. Secondo la tradizione storiografica che fa capo a De Orellana, l'artista che ideò il progetto di riforma rococò del tempio fu Hipólito Rovira, mentre la realizzazione pratica dell'opera fu affidata a Luis Domingo (1967: 471). Da un lato la storiografia, dall'atro la documentazione, almeno quella conosciuta finora, che si basa sui protocolli del notaio Agustín Bonet (González Tornel 2011: 101-103). Essa attesta che il 23 settembre del 1751 la giunta di fabbrica contrattò col mastro muratore Antonio García l'opera di rinnovamento del presbiterio, dei primi due tratti della navata ad esso collaterali e delle rispettive cappelle. García si doveva limitare alla realizzazione delle stuccature «di liscio» ovvero alla preparazione delle superfici perché potessero ricevere la decorazione figurativa e la doratura. Senza ulteriori dettagli su chi effettivamente realizzò gli adorni figurativi, nel dicembre del 1753 si subappaltò al pittore e doratore Félix Lorente il dorato e lustramento dell'opera realizzata fino ad allora che, contrariamente a quanto stipulato precedentemente comprendeva anche il terzo tratto della navata con le rispettive cappelle.

Allo stato attuale della ricerca il contratto con Lorente costituisce l'unica fonte di cui si dispone per conoscere alcune delle tecniche o dei modelli stilistici impiegati nella rinnovazione rococò più importante della città di Valencia. Quanto ai procedimenti, per la doratura si registra l'impiego della corladura, tecnica consistente nell'applicare velature di vernice su superfici metalliche ben lucidate. Il successo di questa tecnica di doratura in area valenziana fu dovuto a una capacità di rifrazione della luce naturale quasi identica a quella dell'oro puro, unita ad un grande risparmio economico (Martínez Hurtado 2002: 140). Per ciò che concerne la lustratura, nel capo ottavo del contratto si stabilì che Lorente impiegasse sottili strati di gesso di ottima qualità e materiali organici come colle distillate, applicati sulle superfici con strumenti caldi o direttamente con le mani. Infine, rispetto ai modelli formali, numerosi furono i riferimenti alle concezioni stilistiche del tempo; molto preciso 
è in particolare il capo numero 9 che cita lo stile tardo-barocco tedesco (Milam Dawn 2011: 37). Il 6 febbraio del 1754 si contrattarono i muratori Juan Simarro e Bautista Pontons per rivestire i piedistalli dei pilastri e contrafforti della zona sino ad allora rinnovata (Pingarrón 1997: 339340, 354-359). Sebbene quest'ultima commessa dovesse concludersi entro il mese di ottobre di quello stesso anno, diverse vicissitudini, tra cui la rescissione del contratto da parte di Simarro e la sopravvenuta morte di Pontons, i pagamenti avvennero soltanto nel 1765, anno in cui il Marchese de Dos Aguas abbonò la somma dovuta agli eredi di Pontons.

La presenza di Giner Rabassa de Perellós nella giunta di fabbrica, che si registra già nella riunione in cui si stabilì di avviare le opere di rinnovamento, diventò determinante dal 1755, anno in cui il Marchese chiese di essere nominato obrero mayor per potersi addossare i costi della riforma. Effettivamente, spesso la riforma del tempio di San Andrés si è considerata come una prosecuzione della rinnovazione del vicino palazzo de Dos Aguas, della quale si è pensato costituisse una sorta di cappella gentilizia. Questo spiegherebbe la ragione per la quale si impiegò la stessa equipe di artisti, o parte di essa, che aveva progettato e posto in essere la riforma del palazzo marchesale (Coll Conesa 2005: 15-16). I protocolli di Bonet proporzionano un ultimo dato documentale che non deve essere sottovalutato, ovvero la presenza del pittore José Vergara nella Giunta di fabbrica a partire dal 1768.

Queste le uniche, poche certezze documentarie per cui si possono soltanto ipotizzare al momento la responsabilità della rinnovazione rococò e le fasi della sua realizzazione. Quello che è certo è che il risultato fu qualcosa di assolutamente innovativo e mai visto prima a Valencia. Al riguardo Elías Tormo ebbe a scrivere (1943: 388):

\begin{abstract}
Un caso único de una modalidad artística del arte rococo de Valencia: una creación de carácter personal, sin repetición conocida, allí, ni fuera de allí. El total interno de San Andrés es una joya de arte decorativo, y es una gloria (sin duplicado, sin repetición) en la Historia del Arte.
\end{abstract}

Effettivamente, l'interiore del tempio di San Andrés sembra essere stato rivestito da una preziosa pelle di porcellana in cui si susseguono rocaille, finti tessuti, rosoni, cornucopie di frutti, ghirlande di fiori, putti e angioletti in forte scorcio, con attitudini e pose tutte differenti. Ma abbondano anche statue allegoriche, scudi e medaglioni, emblemi e geroglifici che dotano l'insieme decorativo di un forte ed inusuale senso iconologico. Il tutto di un intenso bianco lucente, dovuto alla lustratura dello strato più esterno dello stucco, in relazione all'oro del corlado e a superfici che seppur bianche furono volutamente meno definite, per creare suggestivi ed affascinanti effetti di chiaroscuro e giochi di luci ed ombre. In definitiva, si può affermare che per la prima volta a Valencia la decorazione diventa la vera protagonista, causa e motore di un cambiamento senza precedenti: veicolare quei messaggi iconologici la cui espressione un tempo si riservava a Scultura e Pittura, mentre l'adorno si limitava a riempire gli spazi che esse lasciavano vuoti. Non che in San Andrés manchino le pitture, anzi la chiesa offre oggigiorno un fondamentale campionario della pittura valenziana degli anni di passaggio dal tardo-barocco all'Accademismo. Tuttavia, la loro presenza si limita alle cappelle 
laterali, dove hanno l'obiettivo di celebrare il santo che ne detiene la titolarità. Inoltre, non lo fanno da sole, bensì in relazione con i ricchissimi zoccoli ceramici e soprattutto con i numerosi emblemi in stucco che le rivestono, quindi ancora una volta in relazione alla dominante decorazione. Ed è nella controfacciata che il protagonismo decorativo si fa ancora più asfissiante ed esagerato.

Ciò introdusse un ulteriore elemento di novità nel medio artistico valenzano, perché per la prima volta si diede maggiore importanza iconografica alla controfacciata. C'erano, è pur vero, dei precedenti specificamente valenziani. Si pensi alla controfacciata della Chiesa di San Nicola di Bari e San Pietro Martire con la magnifica rappresentazione al fresco dell'allegoria della Chiesa Trionfante o a quella della citata Chiesa de los Santos Juanes che albergava le scene iniziali della vita dei due santi titolari rappresentate negli ovali pittorici e fiancheggiate da statue allegoriche. Ma si trattava comunque pur sempre di isolati casi, nei quali per di più non deve dimenticarsi la fortissima influenza che ambienti culturali diversi, soprattutto di ambito italiano, ebbero nella definizione dei programmi iconografici.

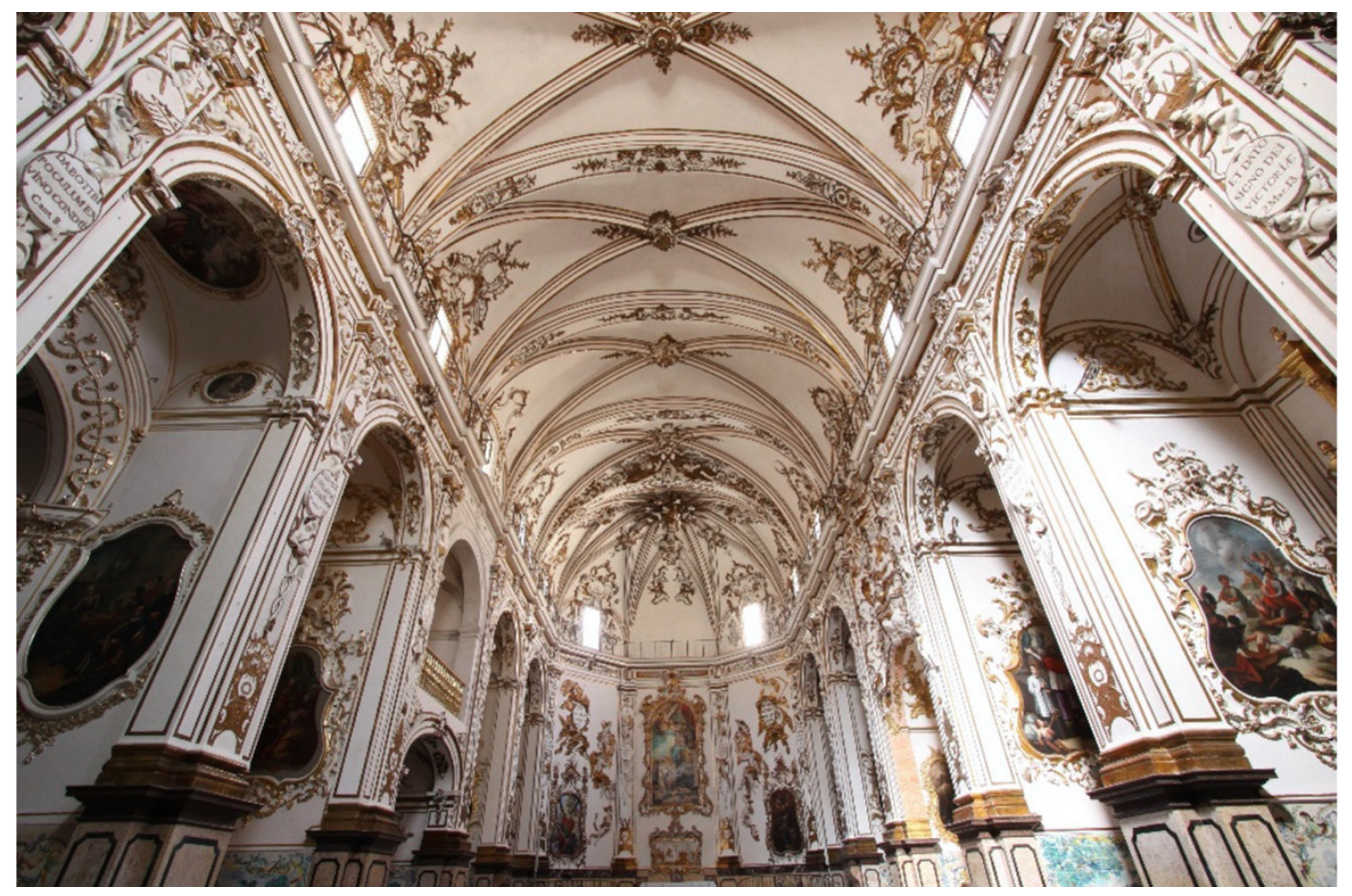

Figura 4 Luis Domingo stuccatore (attr.), Hipólito Rovira progettista (attr.), Interiore della Chiesa di San Andrés, attuale San Juan de la Cruz, Valencia, 1751-1753. Fotografia dell'autore. 
La maestosa controfacciata di San Andrés si configura come una grandissima cortina tesa alle estremità e al centro del fregio e che arriva a lambire il portale di ingresso del tempio e le due nicchie che lo fiancheggiano. Su questo telo, come aperto verso una visione ultraterrena, appaiano numerose figure allegoriche di Ripiana memoria che rappresentano le virtù del santo titolare della chiesa (Figura 5).

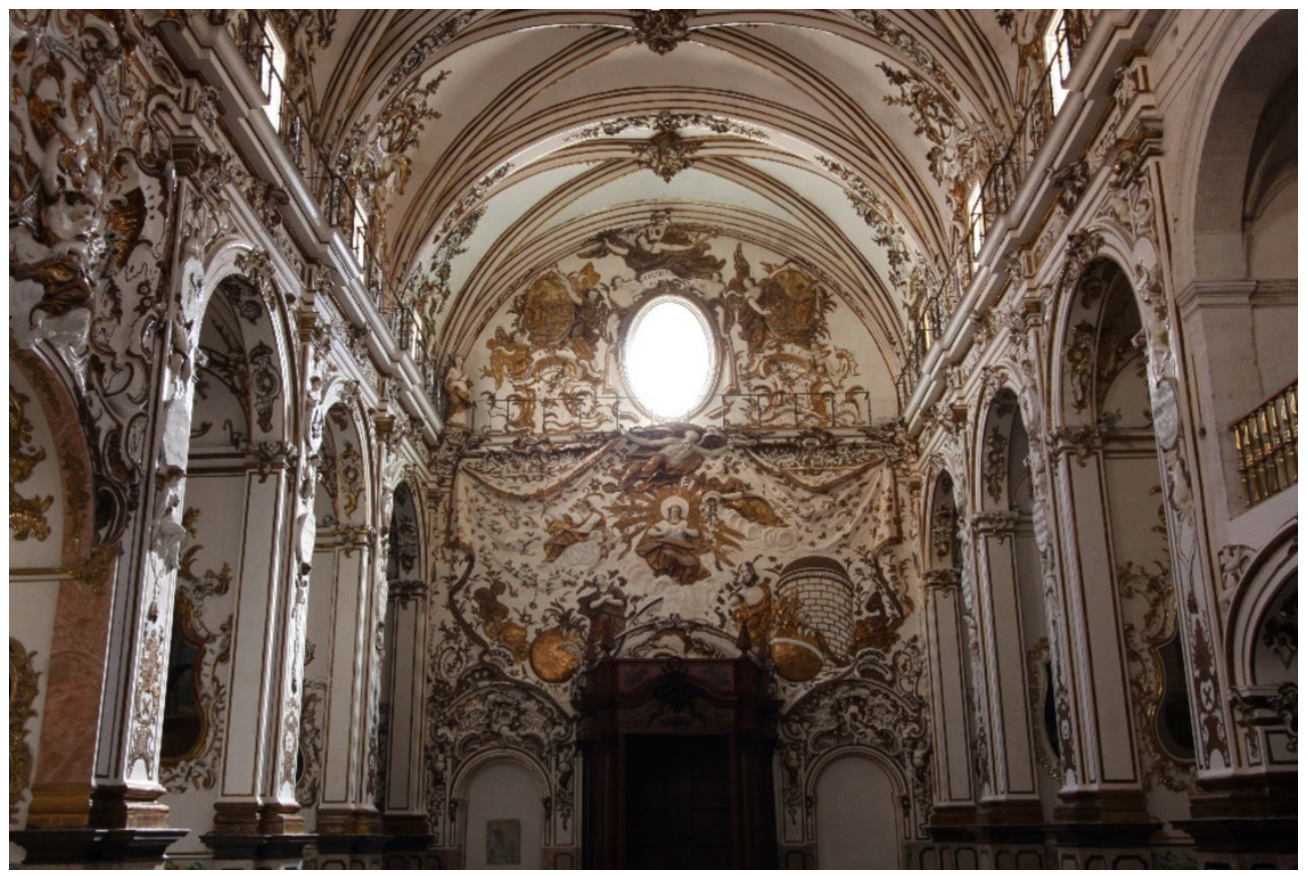

Figura 5 Luis Domingo stuccatore (attr.), Hipólito Rovira progettista (attr.), Controfacciata, Chiesa di San Andrés, attuale San Juan de la Cruz, Valencia, metà del XVIII secolo. Fotografia dell'autore.

Queste sono dominate dalla prominente allegoria del Tempo rappresentato in volo nel vertice centrale della cortina. Sintesi delle varie versioni proporzionate da Ripa, il padre Tempo di San Andrés è rappresentato quale anziano alato che tiene la clessidra nella mano sinistra e la falce, purtroppo perduta nell'attualità, nella destra. Verso la figura del Tempo si dirige in volo un piccolo putto alato che tiene in mano un cerchio dorato, classico simbolo dell'eternità. Al passare del tempo e al suo dominio sulle umane vicende sono connesse anche le due sfere ai piedi della cortina, 
rappresentanti il Sole e la Luna (Ripa 1603: 482-483). La figura femminile centrale è la Benignità rappresentata nell'atto, citato da Ripa, di spremersi i seni per nutrire con carità il prossimo. La veste ricamata con stelle di cui parla il trattatista italiano è sostituita nella controfacciata valenziana da raggi dorati simboleggianti l'origine divina di questa virtù, mentre di altari accesi ce ne sono due, per ovvie ragioni di simmetria tanto care all'estetica rococò (Ripa 1603: 43-44). La Benignità è fiancheggiata, alla sua destra e alla sua sinistra rispettivamente dalla Misericordia e dalla Pietà, virtù innegabilmente connesse tra loro. La Misericordia, distesa su una nube, è identificabile grazie all'attributo del ramo di quercia che tiene con la mano sinistra, posto che mancano gli atri attributi citati da Ripa, ovvero la ghirlanda d'ulivo sul capo e la cornacchia (Ripa 1603: 328-330). Ugualmente semplificata rispetto al trattato Ripiano è l'allegoria della Pietà identificabile esclusivamente per la cornucopia i cui doni riversa abbondanti (Ripa 1603: 401-403). Ai piedi della cortina, inginocchiata sull'arco che sovrasta il portale d'ingresso del tempio, è l'allegoria dell'Umiltà con il viso estatico e le braccia incrociate sul petto (Ripa 1603: 214-216). Sul lato sinistro le corrisponde simmetricamente la Fortezza, ovvero una figura rivestita con armatura ed elmo che tiene accanto una grande torre; questa, che sostituisce la colonna indicata invece da Ripa, solitamente si attribuiva alla Fermezza (Ripa 1603: 156, 165-169).

Le allegorie sin qui analizzate sono immerse in uno spazio ricco di fiori e altri elementi decorativi vegetali dorati che contrastano sullo sfondo bianco. Il senso iconografico finalizzato, come si diceva, all'esaltazione delle virtù e del valore esemplare della vita di Sant'Andrea continua nella rappresentazione della Chiamata all'apostolato dei Santi Andrea e Pietro e di Gesù che cammina sulle acque nei medaglioni in stucco dorato della lunetta, sorretti da elegantissimi angeli. Questi, insieme a rocaille e ornamenti vegetali naturalistici, fiancheggiano una finestra ovale coronata, infine, dalla figura allegorica della Fama in volo, che ricorda molto quella scolpita da Ignacio Vergara nel portale del Palazzo de Dos Aguas.

La controfacciata non è l'unico spazio nel quale si sviluppa il messaggio iconografico del tempio valenzano di San Andrés. Basti citare i numerosissimi emblemi che rivestono i contrafforti tra le cappelle, arricchiti da citazioni bibliche in latino e scudi con la croce di Sant'Andrea e la corona di spine alternativamente. O ancora, la delirante decorazione dell'arco di accesso alla distaccata cappella di San Giovanni Nepomuceno con la rappresentazione di Sansone che raccoglie il miele dalla carcassa del leone, chiaro riferimento alla virtù di saper mantenere il segreto della confessione per la quale il santo ceco fu martirizzato (Figura 6). 


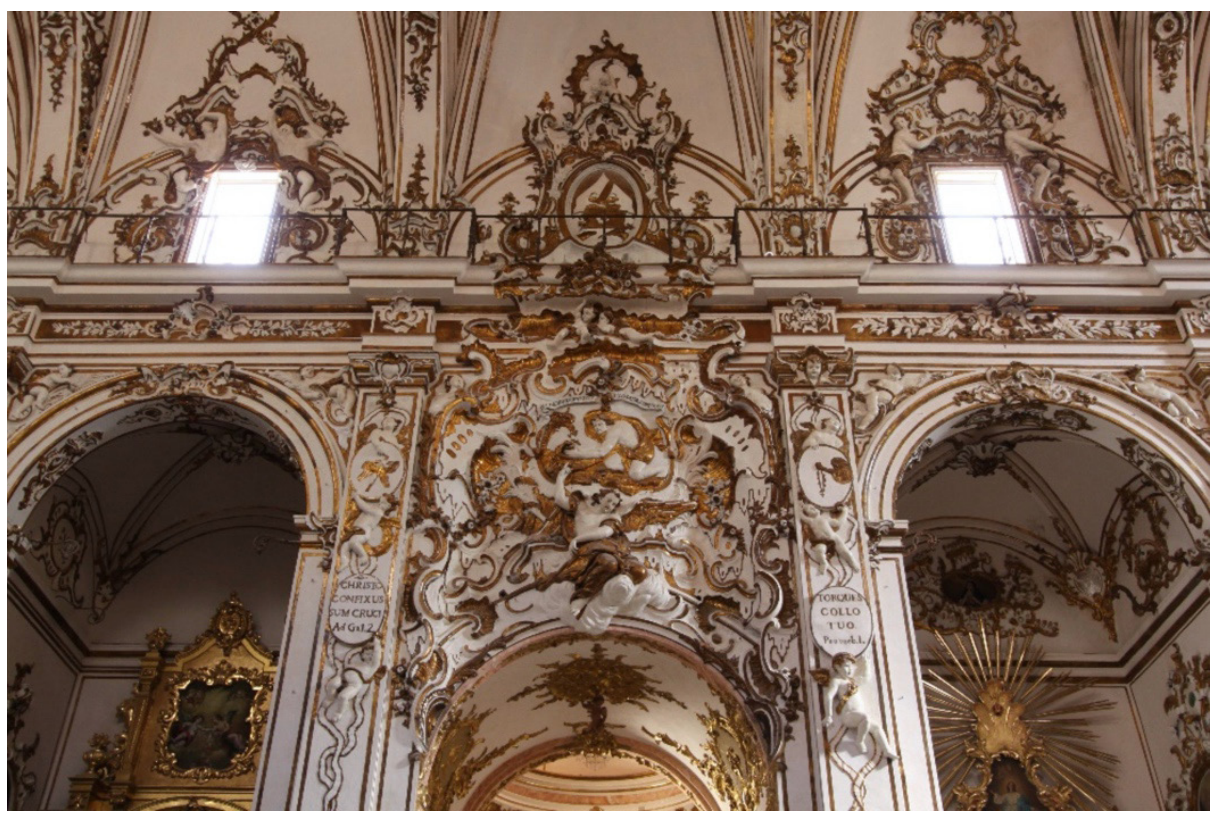

Figura 6 Luis Domingo stuccatore (attr.), Félix Lorente doratore, Hipólito Rovira progettista (attr.), Arco d'accesso alla Cappella di San Giovanni Nepomuceno, Chiesa di San Andrés, attuale San Juan de la Cruz, Valencia, 1751-1753. Fotografia dell'autore.

Ciò che deve sottolinearsi ancora una volta è che questa funzione iconologica venne assolta per la prima volta completamente dalla ricca ed esuberante decorazione in stucco, campionario completo del più tipico linguaggio rococò fatto di cartouches asimmetrici, curve e controcurve, shellworks, ali di pipistrello, palmette, forme fantastiche, esotiche ed innaturali (Milam Dawn 2011: 23-24). Ma tanto per i testimoni contemporanei alla realizzazione dell'opera, quanto per Tormo l'elemento più distaccabile dell'intera riforma rococò del tempio fu il pulpito, definito «lo más interesante del rococó españob) (De Orellana 1967: 471; Tomo y Monzó 1923: 107). Disgraziatamente distrutto durante la Guerra civile, era opera attribuita a Luis Domingo, che si trovava addossata al contrafforte tra la cappella di San Giovanni Nepomuceno e quella dei Santi Bonaventura e Tommaso d'Aquino. Su una elegante rocaille bordata d'oro si trovava l'imponente figura di un angelo, presumibilmente dorato anch'esso, rappresentato nello sforzo di sorreggere la cassa del pulpito. Era questa di pianta sinuosamente polilobata, intarsiata e policromata; vi si distinguevano tre scudi monocromi, due laterali ed uno frontale, con scene bibliche purtroppo non riconoscibili ma presumibilmente legate al tema della predicazione. Egualmente policromo era lo schienale, con eleganti motivi rococò, curve e controcurve ad « $S »$ e $« \mathrm{C} »$. Il tutto era ricoperto da una pensilina che riprendeva le linee della 
cassa e da cui pendevano falde di finto tessuto; dominavano l'insieme una scultura della Fama in atto di suonare la tromba e un rosone di raggi dorati.

\title{
5. La reazione al Rococò e le sue infauste conseguenze
}

Le maggiori opere del Rococò valenzano, che precedentemente si sono brevemente analizzate, furono realizzate in un periodo di pieno fermento culturale. Dopo il tentativo fallito dell'Accademia di Santa Barbara, il 14 febbraio del 1768 venne fondata la Real Academia de Bellas Artes de San Carlos de Valencia. Già nel momento della sua istituzione, i suoi membri furono chiari nell'esprimere la volontà di controllare la produzione artistica della città. In particolare, si voleva impedire che si perpetuasse ancora l'uso settecentesco di scultori e pittori che si intromettevano nel fare architettonico. A detta dei professori della nascente Accademia, il mal gusto era stato introdotto nell'Arte proprio da quegli artisti che, privi di qualunque conoscenza scientifica sulle basi dell'Architettura, ugualmente si arrischiavano a praticarla. Si legge nello statuto quanto segue (1828: LV-LX):

\begin{abstract}
Para remediar los abusos y desórdenes que la ignorancia y menosprecio de las artes ha ido insensiblemente introduciendo en prejuicio de la misma Ciudad de Valencia, y de sus vecinos: [...] prohíbo á todo Tribunal, Ministro, Juez ó Gremio que hasta ahora haya dado título ó facultad para tasar, medir, idear y dirigir fábricas, la continuación de darlos sin que proceda examen y aprobación del pretendiente en la Academia. Cualquier título que sin estas circunstancias se conceda lo declaro nulo y de ningún valor [...] Es mi voluntad que todos los que desde el presente día en adelante hayan de ejercer la arquitectura, y señaladamente el medir, tasar, idear y dirigir fábricas, han de ser precisamente habilitados por la Academia y no por otro Tribunal, Magistrado, Gremio, ni persona alguna precediendo un riguroso examen hecho en Junta ordinaria, no solo de la teórica de la Arquitectura, sino también de la práctica de la Geometría, Aritmética, Maquinaria, y demás ciencias matemáticas necesarias para hacer con acierto unas operaciones en que tanto se interesan mi vasallos [...] Prohíbo muy expresamente á todas las Juntas, Congregaciones, Gremios, ú otras cualesquiera Comunidades y personas que hay y pueda haber en Valencia, que ahora ni en ningún tiempo se puedan mezclar, ni entrometer con motivo alguno en el estudio, práctica, ni arreglo de las Nobles Artes Pintura, Escultura, Arquitectura y Grabadura, bajo la pena á quien lo contrario hiciere de cien ducados por la primera vez, doscientos por la segunda, y trescientos por la tercera: declarando como declaro, que el estudio y la práctica de las dichas Nobles Artes es cuidado propio y privativo de la Academia, sin que por nadie se le pueda turbar en él.
\end{abstract}

Non si fece aspettare la reazione degli artisti, talvolta sostenuti ed incitati anche da membri dell'Accademia, i fratelli Vergara in testa, la cui formazione era avvenuta nel clima poliedrico del Barocco. Questo diede vita ad una querelle che si concluse solamente nel 1778, dopo che ognuna delle sezioni dell'Accademia presentò le ragioni per cui riteneva fosse suo diritto controllare la produzione della decorazione architettonica. Nella relazione emessa dai professori della sezione di Architettura si legge: ${ }^{1}$

1 Archivio della Real Accademia di San Carlos (in seguito ARASC), fasc. 67-A/105, ff. 5a-5b. 


\begin{abstract}
El mismo se ha engendrado de la corrupción de las artes, y se ha propagado y extendido en detrimento y ruina de las mismas, destruyéndose por ese camino la simplicidad de la buena arquitectura, y desterrando el adorno verdadero, causando fealdad y confusión a la vista, como se nota en la decoración de algunos edificios que de un tiempo acá se han construido y renovado, ya sean sagrados o profanos, privados o públicos. No se puede mirar sin dolor las iglesias de san Martín, santa Catalina y san Andrés, echada a perder la buena arquitectura de esta última por un escultor cuyo buen nombre es la estatuaria merece la veneración de los profesores de su arte, pero erró en esta parte por haverse mezclado en lo que no era su inspección.
\end{abstract}

Dure furono anche le parole di Pedro de Silva, accademico di merito della Corte, chiamato a Valencia per risolvere definitivamente la questione in esame e che espresse quanto segue: ${ }^{2}$

\begin{abstract}
A menos que se quiera hacer una obra bárbara y digna de desprecio, como el famoso púlpito de la parroquia de san Andrés que está circuido de unas pilastras desproporcionadas, y unos bazos relieves mal colocados; y sostenido sobre un angelón, que en toda buena regla debe venir al suelo, porque no tiene más apoyo que el de una cartela que sale menos de un palmo del muro, lo qual repugna a la firmeza aparente que debe consultarse en todas las obras.
\end{abstract}

Pertanto, il nascente Accademismo di matrice neoclassica condannò la decorazione rococò della Chiesa di San Andrés come esempio, tra altri, della degenerazione e corruzione delle Arti, di cui gli scultori e pittori che si intromettevano nelle commesse architettoniche erano promotori e che la nascente istituzione valenciana aveva il dovere di combattere (Bérchez 1987: 183-216). L'Accademia valenciana poi non fu l'unica a condannare gli esuberanti stucchi e le deliranti allegorie di San Andrés. Dure, ad esempio, furono anche le parole di Antonio Ponz (1725-1792), pittore ed intellettuale esemplificativo della nuova corrente neoclassicista accademista, che nella cronaca del suo monumentale Viaje de España criticò le modernizzazioni attuate nel tempio dedicando alla sua descrizione poche scarne righe (1988: 711).

Certo è che per circa due secoli si cercò di dimenticare quanto a Valencia fosse stato prodotto durante il Barocco e soprattutto nella sua fase tarda. Infatti, prima il Neoclassicismo accademista lo condannò esortando ad un ritorno all'ordine e al decoro degli ordini classici, poi, durante l'Ottocento, il Romanticismo e i movimenti nazionalisti premettero per il recupero delle antichità medievali e gotiche della città, che secondo le loro concezioni meglio esprimevano l'originalità culturale valenziana (Garín Llombart - Pons Alós 2009: 26-27). Come si è accennato, tra il 1862 e il 1867, il Palazzo de Dos Aguas fu rinnovato quasi nella sua interezza secondo i neostili eclettici tanto in voga in quel periodo (Coll Conesa 2005: 107-113). Attualmente è sede del Museo Nazionale di Ceramica e i lavori novecenteschi per l'allestimento museale hanno vieppiù contribuito a occultare la precedente riforma rococò. Il vicino tempio di San Andrés venne quasi dimenticato, se si esclude un

2 ARASC, Libro I, «Acuerdos en limpio de Juntas particulares desde el año 1765 hasta el año 1786» (la relazione di Pedro de Silva si trova nella pagine successiva all'inizio della Giunta particolate del 24 maggio e ocupa le Quattro pagine che le seguono). 
urgente intervento di restauro di fine Ottocento dovuto a ingenti infiltrazioni d'acqua che stavano causando il disfacimento dello strato decorativo (Tormo Esteve - Ordaz Benat 2010: 215-233). Lo stato delle cose era tanto pessimo che nel 1902 si decise di spostare la titolarità della chiesa ad un edificio moderno della Calle Colón, dove si trasferirono anche preziosi beni mobili. La Guerra civile non migliorò la situazione, causando come si accennava la distruzione del pulpito, dell'organo e del retablo, causando danni seri agli stucchi e alle ceramiche, ricoprendo di fumi e di polvere le pitture e lo strato decorativo. Per questo suo degrado ed essendo stato privato del permesso di svolgervi culto, si decise di abbattere il tempio, insieme ad altri della città che versavano nelle stesse disperate condizioni, come San Bartolomeo e San Michele e Dionisio. Il grande successo che San Andrés non abbia subito lo stesso triste destino delle citate chiese e che ancora oggi, dopo i restauri del 2009, possa mostrarsi ai visitatori in tutto il suo delirante splendore si deve a Elias Tormo y Monzó (Garín Llombart - Pons Alós 2009: 497). Questi, contrastando con agguerriti articoli di stampa il Municipio di Valencia, l'Accademia di San Carlos e persino l'Arcivescovato, conseguì che la chiesa fosse dichiarata Monumento Nazionale nell'aprile del 1942 e che fosse riaperta al culto in seguito all'istallazione in essa del Convento carmelitano di San Juan de la Cru\%:

\section{Conclusioni}

In questo contributo si è voluto dare rilevanza a quei congiunti artistici e singole opere che nella storiografia e trattatistica di Sette, Otto e Novecento sono stati enumerati tra i pochissimi esempi del Rococò nella città di Valencia. Seguendo le tracce lasciate da Orellana, Ponz o Tormo si è tentato quindi di realizzare uno studio organico e complesso della fase rococò della evoluzione artistica valenziana, senza che esso pretenda di essere esaustivo e completo, soprattutto a causa dei grandi vuoti documentali che ancora si registrano e soprattutto della perdita di gran parte dei monumenti analizzati. Si è tentato di confutare l'idea implicita nelle parole dei citati trattatisti che quelle opere fossero solo casi sporadici, meteoriche eccezioni, incapaci di condizionare in qualche modo l'evoluzione della produzione artistica valenziana all'alba del Neoclassicismo. Ci si auspica, in conclusione, che queste pagine possano contribuire alla riscoperta di un periodo della Storia dell'Arte della città di Valencia che ha lasciato non solo delle casuali eccezioni, bensì invece alcuni gioielli architettonici, scultorei, pittorici, decorativi unici e irripetibili; che possa portare a rivalutare artisti per troppo tempo considerati secondari o minori, quasi schiacciati dalle pretese dell'Accademismo che stava sorgendo; artisti che, con la loro arte e con la loro abilità nel mischiare e relazionare le tecniche e le conoscenza acquisite, hanno saputo cogliere il senso estetico della società in cui operavano e tradurlo in capolavori che oggi è necessario rivalutare; $\mathrm{e}$ in ultima istanza che consenta di aprire nuovi orizzonti di conoscenza verso quella società, una società nuova e in continua mutazione, che abbandonava i rigidi schemi locali e si apriva ad istanze culturali e quindi artistiche ormai europee. 


\section{Bibliografia}

Aldana Fernández S. (1976) «La portada del Palacio de los marqueses de Dos Aguas», Trazay Baza, 6, pp. 89-97.

Bérchez, J. (1983), a cura di, Catálogo de monumentos y conjuntos de la Comunidad Valenciana, Valencia, Conselleria de Cultura, Educación y Ciencia de la Generalitat Valenciana, 2 voll.

—_. (1987) Arquitectura y academicismo, Valencia, Institución Alfonso el Magnánimo.

—_. (1993) Arquitectura barroca valenciana, Valencia, Bancaja.

- (1995) a cura di, Monumentos de la Comunidad Valenciana. Valencia Arquitectura Religiosa, tomo X, Valencia, Generalitat Valenciana.

Breve Noticia de los principios, y progresos de la Academia de Pintura, Escultura, y Arquitectura; erigida en la ciudad de Valencia baxo el título de Sant Bárbara; y de la proporción que tienen sus naturales para estas Bellas Artes (1757) Madrid, Gabriel Ramírez.

Buchón Cuevas, A. Ma . (2012) «Pintar la escultura: apuntes sobre doradores del siglo XVII y XVIII en Valencia», Ars Longa: cuadernos de arte, 21, pp. 197-214.

Colección de Reales Ordenes comunicadas a la Real Academia de San Carlos, desde el año de 1770 hasta el de 1828 (1828) Valencia, Benito Monfort.

Coll Conesa, J. (2005) a cura di, Elpatrimonio artístico e histórico de los Rebassa de Perellós y el Palacio de Dos Aguas, Valencia, Amigos del Museo Nacional de Cerámica y Artes Suntuarias González Martí.

De Orellana, M. A. (1967 [1 ${ }^{\mathrm{a}}$ ed. fine del XVIII secolo]), Biografía pictórica valentina o Vida de los pintores, arquitectos, escultores y grabadores valencianos, Valencia, Ayuntamiento.

Estatutos de la Real Academia de San Carlos (1828) Valencia, Benito Monfort.

Ferrer Orts, A. (2000) «Sobre la decoración esgrafiada en el barroco español», Ars Longa: cuadernos de arte, 9-10, pp. 105-109.

Garín Llombart, F. V., Pons Alós, V. (2009) a cura di, La Gloria del Barroco. Valencia 2009-10. Catálogo de la exposición, Valencia, Generalitat Valenciana.

Gómez-Ferrer Lozano, M. (1995) «La antigua iglesia parroquial de San Andrés de Valencia y la arquitectura valenciana en la transición al siglo XVII», Boletín de la Real Academia de Bellas Artes de San Fernando, 80, pp. 235-258.

González Tornel, P. (2005a) Arte y arquitectura en la Valencia de 1700, Valencia, IAM. . (2005b) «Francisco Vergara y los revestimientos arquitectónicos del barroco valenciano en las primeras décadas del siglo XVIII», Archivo de Arte Valenciano, 86, pp. 41-51. 
González Tornel, P. (2008) «Barroquizar la arquitectura. Intervenciones de signo barroco en construcciones eclesiásticas medievales de la ciudad de Valencia», in Historia de la ciudad. V. Tradición y progreso, Valencia, Colegio Oficial de Arquitectos de la Comunidad Valenciana, pp. 131-152.

(2011) «El ornamento arquitectónico como base del cambio de gusto en la Valencia de mediados del siglo XVIII. De los estucos de la Parroquia de San Andrés a los modelos académicos de Vicente Gascó en la Capilla del Carmen», Ars Longa: cuadernos de arte, 20, pp. 97-108.

Martínez Hurtado, S. (2002) «El dorado. Técnicas, procedimientos y materiales», Ars Longa: cuadernos de arte, 11, pp. 137-142.

Milam Dawn, J. (2011) Historical dictionary of Rococo art, Lanham, Scarecrow Press.

Pérez Guillén V. I. (2008) «La portada de Dos Aguas: Bernini, Ripa, Rovira. La huella de Bernini en la portada del palacio del Marqués de Dos Aguas», Archivo de Arte Valenciano, 89, pp. 65-80.

Pingarrón, F. (1997) «Algunos documentos sobre las reformas tardobarrocas de las iglesias de San Andrés, Santa Catalina y San Martín en Valencia a mediados del siglo XVIII», Saitabi: revista de la Facultat de Geografia i Història, 47, pp. 327-363.

-. (1998) Arquitectura religiosa del siglo XVII en Valencia, Valencia, Ayuntamiento.

Ponz, A. (1988 [1ª ed. 1772-1794]) Viaje de España, vol. I, tomi I-IV, Madrid, Aguilar.

Ripa, C. (1603) Iconologia overo Descrittione di diverse imagini cavate dall'antichità, \& di propria inventione, trovate \& dichiarate da Cesare Ripa Perugino, Cavaliere de' Santi Mauritio \& Lazaro. Di nuovo revista, \& dal medesimo apliata di 400 \& più imagini. Et di figure di intaglio adornata. Opera non meno utile che necessaria a Poeti, Pittori, Scultori, \& altri, per rappresentare le Virtù, Vitij, Affetti, \& Passioni bumane. Roma, Lepido Facij.

Sebastián López S. (1989) «Nueva lectura iconográfica-iconológica de la portada del Palacio del marqués de Dos Aguas», Goya: revista de arte, 211-212, pp. 60-66.

Tormo Esteve, S. - Ordaz Benat, C. (2010) La Gloria del Barroco: campaña de restauración patrimonial, Valencia, Generalitat Valenciana.

Tormo y Monzó, E. (1920a) «El arte barroco en Valencia», Arte español, tomo V, numero 3, pp. 114-129.

- (1920b) «El arte barroco en Valencia», Arte español, tomo V, numero 4, pp. 174-188.

—. (1921) «El arte barroco en Valencia», Arte español, tomo V, numero 5, pp. 201-212.

- (1923) Levante: provincias valencianas y murcianas, Madrid, Calpe.

—_. (1943) «Cuatro más de los monumentos de la ciudad de Valencia en peligro de pérdida: iglesia de San Andrés de Valencia; el jardín de Monforte en la ciudad de Valencia; el Palacio de Dos Aguas en Valencia; San Juan del Hospital de Valencia», Boletin de la Real Academia de la Historia, tomo CXIII, cuaderno 2, pp. 385-414.

Vilaplana Zurita, D. (1997) Programas iconográficos en el arte valenciano del siglo XVIII, Valencia, Universitat de València, 3 vol. (tesi dottorale). 